\title{
Stress-assist Chemical Reactions Front Propagation in Deformable Solids
}

\author{
Alexander Freidin ${ }^{a}$, Elena Vilchevskaya ${ }^{b}$ \\ Institute for Problems in Mechanical Engineering \\ Russian Academy of Sciences \\ Bolshoy pr., 61, V.O. St. Petersburg, 199178 Russia R \\ a alexander.freidin@gmail.com, ${ }^{b}$ vilchevska@gmail.com
}

Keywords: Chemical affinity tensor, Eshelby stress tensor, configurational forces, reaction front kinetics, stress state, mechanochemistry, axially-symmetric problems.

\begin{abstract}
We consider a stress-assist chemical reaction front propagation implying the reaction like silicon oxidation. We assume that the chemical reaction is localized at the reaction front that divides two solid constituents. The reaction is sustained and controlled by the diffusion of the gas constituent through the oxide. We determine a transformation strain tensor produced by the chemical reaction in dependence on the reaction parameters. Then we derive an expression of the entropy production due to the reaction front propagation and, as a result, obtain the formula of the chemical affinity tensor. The normal component of the chemical affinity tensor acts as a configurational force that drives the propagating reaction front. Then we introduce the notion of the equilibrium gas constituent concentration as the concentration at which the chemical affinity is zero. We formulate a kinetic relationship for the reaction front velocity in terms of current gas concentration at the reaction front and the equilibrium concentration that depends on stresses at the front. We obtain analytical solutions of simplest axially symmetric problems of mechanochemistry considering chemical reactions around a hole as a simplest stress concentrator and the oxidation of a cylinder. We demonstrate the reaction locking effects due to internal stresses and examine how stress state affects the reaction front kinetics.
\end{abstract}

\section{Introduction}

The influence of mechanical loading on chemical reaction kinetics remains to be of significant interest for both fundamental and applied engineering science. Chemo-mechanical problems have received a new attention in recent years due to miniaturization of structure elements. For example, fracture processes in micron-scale parts of MEMS made of polycrystalline silicon thin films involve sequential oxidation of polysilicon and environmentally-assisted crack growth inside an oxide layer. In turn, the kinetics of the oxide growth is determined by mechanical stresses produced by the crack. The catastrophic failure happens when the crack reaches the reaction front. Thus, major events which determine the life time of MEMS are related with coupling between stresses and chemical reactions (see details in $[1,2]$ ). Reactions similar to the silicon oxidation also take place in the process of metal hydride formation used in hydrogen storage applications (see e.g. [3]).

Many models of silicon oxidation arise to pioneering papers by Deal and Grove [4], see for example a recent paper [3]. However neither external loading nor internal stresses were taken into account in these works.

One of the first attempts to obtain an expression of the chemical potential in a multicomponent solid under stress was made by Larche and Cahn [5-8]. They considered diffusing solids and showed that the chemical potential depends on the trace of the stress tensor. This result was further developed in 
[9] where it was justified that the chemical potential is proportional to the trace of the Eshelby stress tensor.

In the present paper we consider a chemical reaction in an elastic body within the frameworks of configuration forces concept. We derive an expression of the entropy production due to quasi-static propagation of the chemical reaction front. As a result we obtain an expression of the chemical affinity tensor as a combination of Eshelby stress tensors of the solid constituents and a chemical potential of a gas constituent [10]. We formulate a kinetic equation that relates the reaction front velocity and the normal component of the chemical affinity tensor. As a result the front velocity depends on chemical parameters and temperature as well as on stresses. In turn, stresses depend on the position of the reaction front.

Then we introduce the notion of the equilibrium concentration of the gas constituent. We say that the gas concentration is equilibrium at the reaction front if, given temperature, front position and stresses, the chemical affinity is equal to zero. This allows us to formulate a kinetic relationship for the reaction front velocity in terms of current gas concentration at the reaction front and equilibrium concentration that depends on stresses at the front.

If the equilibrium concentration at the reaction front is greater than the concentration at the outer surface of a body then the reaction front propagation is impossible. This observation leads to the possibility of locking effects related with reaction blocking by internal or/and external stresses.

Finally we give solutions of two simplest axially-symmetric mechano-chemical problems (oxidation of infinite body with a cylindrical hole and oxidation of a cylinder) and demonstrate an example of numerical simulation of oxide zone evolution in a specimen with a groove.

\section{Kinematics and constitutive equations}

We assume that the reaction is localized at the reaction front and sustained by the diffusion of the gas constituent through the oxide layer. For simplicity sake we assume that diffusive gas does not produce stresses and strains in oxide, i.e. the oxide is a solid skeleton for the gas constituent, but we take into account that the chemical reaction is accompanied by volume increase. This induces stresses at the reaction front which in turn affects the reaction rate.

Let $\mathrm{v}_{t}=\mathrm{v}_{-} \cup \mathrm{v}_{+}$is a current configuration of a body at time $\mathrm{t}$, and volumes $\mathrm{v}_{-}$and $\mathrm{v}_{+}$are occupied by solid materials $B_{-}$and $B_{+}$, respectively (Fig. 1), and $B_{+}$is produced as a result of a chemical reaction between $B_{-}$and gas constituent $B_{*}: v_{-} B_{-}+v_{*} B_{*} \rightarrow v_{+} B_{+}$, where $v_{-}, \quad v_{*}$ and $v_{+}$are stoichiometric coefficients.

Along with the reference configuration $\mathrm{V}_{0}=\mathrm{V}_{-} \cup \mathrm{V}_{+}$of the material before the reaction we introduce an intermediate reference configuration $V_{g}$ of the body that corresponds to a stress-free oxide (Fig. 1). Material segments $d \mathbf{x}_{-}$and $d \mathbf{x}_{+}$from $\mathrm{v}_{-}$and $\mathrm{v}_{+}$can be related with corresponding segments $d \mathbf{X}_{-}$and $d \mathbf{X}_{g}$ from $\mathrm{V}_{-}$and $\mathrm{V}_{g}$ by elastic deformation gradients:

$d \mathbf{x}_{-}=\mathbf{F}_{-}^{e} \cdot d \mathbf{X}_{-}, \quad \operatorname{det} \mathbf{F}_{-}^{e}=\frac{d v_{-}}{d V_{-}}=\frac{\rho_{0}}{\rho_{-}}, \quad d \mathbf{x}_{+}=\mathbf{F}_{+}^{e} \cdot d \mathbf{X}_{g}, \quad \operatorname{det} \mathbf{F}_{+}^{e}=\frac{d \mathrm{v}_{+}}{d \mathrm{~V}_{g}}=\frac{\rho_{g}}{\rho_{+}}$

where $\rho_{0}, \rho_{-}, \rho_{+}$and $\rho_{g}$ are densities of initial material and oxide in corresponding configurations, and material segments $d \mathbf{X}_{-}$and $d \mathbf{X}_{g}$ are related by the chemical transformations strain tensor $\mathbf{G}[10]$, 
$d \mathbf{X}_{g}=\mathbf{G} \cdot d \mathbf{X}_{-}, \quad \mathbf{G}=g \mathbf{E}, \quad \operatorname{det} \mathbf{G}=\frac{d V_{g}}{d V_{0}}=\frac{\nu_{+} M_{+} \rho_{0}}{v_{-} M_{-} \rho_{g}}, \quad g=\left(\frac{v_{+} M_{+} \rho_{0}}{v_{-} M_{-} \rho_{g}}\right)^{1 / 3}$

$M_{-}$and $M_{+}$are molar masses of solid constituents, $\mathbf{E}$ is the unit tensor.

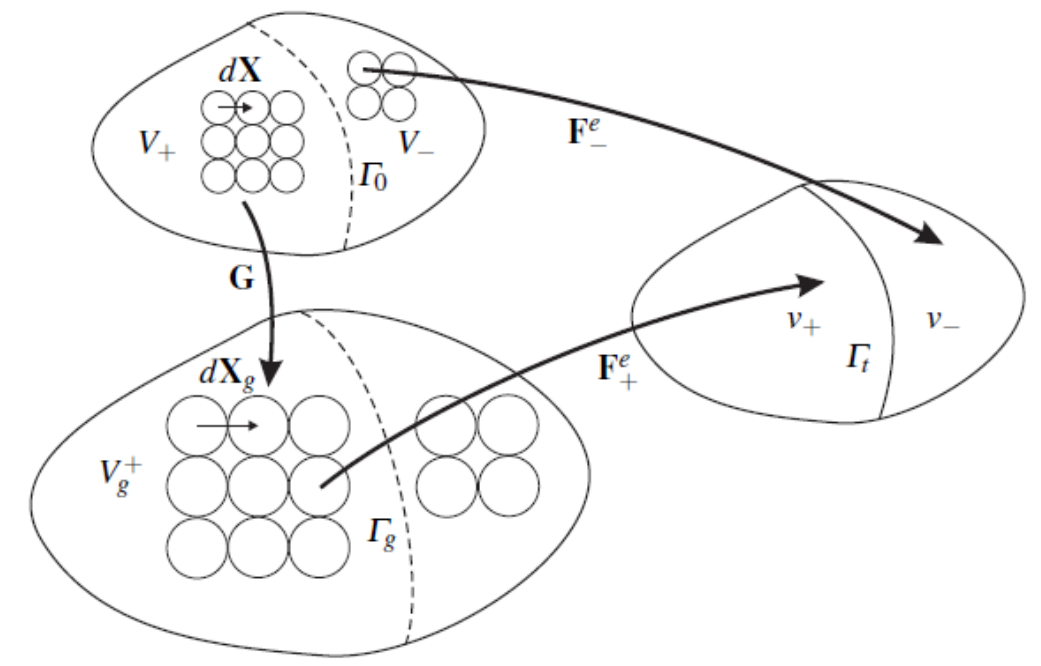

Fig.1. Configurations resulting from chemical transformation and deformation.

A solid skeleton approach leads to the following constitutive equation

$\mathbf{S}_{-}=\rho_{0} \frac{\partial f_{-}}{\partial \mathbf{F}_{-}^{e}}, \quad \mathbf{S}_{+}=\rho_{0} \frac{\partial f_{-}}{\partial \mathbf{F}_{+}^{e}}$

where $\mathbf{S}_{-}$and $\mathbf{S}_{+}$are Piola stresses,

$f_{-}=f_{-}\left(\mathbf{F}_{-}^{e}, T\right), \quad f_{+}=f_{+}\left(\mathbf{F}_{+}^{e}, T\right)$,

are free energy densities, $T$ is the temperature.

\section{Chemical affinity tensor}

The entropy production due to stress-assist chemical reaction front propagation is given by [10]

$T P[S]=\frac{\rho_{0}}{v_{-} M_{-}} \int_{\Gamma} \mathbf{N} \cdot \mathbf{A} \cdot \mathbf{V}^{\Gamma} d \Gamma=\frac{\rho_{0}}{v_{-} M_{-}} \int_{\Gamma} A_{N} V_{N}^{\Gamma} d \Gamma \geq 0, \quad A_{N}=\mathbf{N} \cdot \mathbf{A} \cdot \mathbf{N}, \quad V_{N}^{\Gamma}=\mathbf{V}^{\Gamma} \cdot \mathbf{N}$

where $\mathbf{V}^{\Gamma}$ and $\mathbf{N}$ are a reaction front velocity and a normal to the reaction front with respect to the reference configuration, $\mathbf{A}$ is a chemical affinity tensor,

$$
\mathbf{A}=v_{-} M_{-} \tilde{\mathbf{M}}_{-}+v_{*} M_{*} \tilde{\mathbf{M}}_{*}-v_{+} M_{+} \tilde{\mathbf{M}}_{+}, \quad \tilde{\mathbf{M}}_{+}=f_{+} \mathbf{E}-\frac{1}{\rho_{g}}\left(\mathbf{S}_{+}^{g}\right)^{T} \cdot \mathbf{F}_{+}^{e}, \quad \tilde{\mathbf{M}}_{-}=f_{-} \mathbf{E}-\frac{1}{\rho_{0}} \mathbf{S}_{-}^{T} \cdot \mathbf{F}_{-}^{e}
$$


$M_{*}$ is the molar mass of the gas, $\tilde{\mathbf{M}}_{-}, \tilde{\mathbf{M}}_{+}$, are Eshelby stress tensors determined with respect to $V_{0}$ and $V_{g}$ configurations, $\tilde{\mathbf{M}}_{*}=\mu_{*}(c, T) \mathbf{E}, \mu_{*}$ is a chemical potential of a gas constituent, $c=\rho_{*} / \rho_{g}$ is a relative gas concentration.

Tensorial character of the chemical affinity reflects the fact that the reaction front velocity depends on the orientation of the front with respect to the stress tensor.

\section{The equilibrium gas constituent concentration and kinetic equation}

Basing on the expression of the entropy production (1) one can represent the kinetic equation that determines the normal component of the reaction front velocity as a function of the normal component of the affinity tensor:

$$
V_{N}^{\Gamma}=L\left(A_{N}\right), \quad V_{N}^{\Gamma} L\left(A_{N}\right) \geq 0
$$

Note that the transformation strains and changes in elastic modules lead to internal stresses which in turn affect the chemical reactions front kinetics.

Following [11] and taking into account that the reaction is localized at the reaction front we accept that the reaction rate $\omega$ equal to the number of oxide moles produced in unit time per unit area is given by

$\omega=\hat{\omega}\left(1-\exp \left(-\frac{A_{N}}{R T}\right)\right)$

where $\hat{\omega}=k_{*} c_{-} c \cong k_{*} c$ is a rate of the direct chemical reaction, $k_{*}$ is a kinetic constant, $c_{-}$and $c$ are partial concentrations of reacting constituents, and since the "--" constituent is solid, one can put $c_{-}=1$.

Then, taking into account the mass balance, one can rewrite the kinetic equation (2) as

$$
V_{N}^{\Gamma}=\frac{v_{+} M_{+}}{\rho_{g}} k_{*} c\left(1-\exp \left(-\frac{A_{N}}{R T}\right)\right)
$$

In the case of chemical equilibrium $A_{N}=0$ and the chemical reaction rate is equals to zero. One can introduce a notion of an equilibrium gas concentration at the reaction front as the concentration $c_{e q}$ such that

$$
A_{N}\left(c_{e q}\right)=0
$$

Under accepted assumptions the chemical affinity tensor depends on the gas concentration only through the dependence $\mu_{*}\left(c_{e q}, T\right)$. Then the equation (5) determines the equilibrium concentration in dependence on stresses at the reaction front, temperature and material parameters.

If the equilibrium concentration is found then the current value of the normal component of the chemical affinity tensor can be calculated as

$$
A_{N}=v_{*}\left(\mu_{*}(c(\Gamma), T)-\mu_{*}\left(c_{e q}, T\right)\right)
$$


As a result the reaction front velocity can be calculated by formulae (4), (6) where the equilibrium concentration $c_{e q}$ is to be found from the equation (5). Note that if stresses depend on the front position then $c_{e q}$ also depends on the front position.

Not far from the chemical equilibrium

$$
A_{N}=\left.v_{*} \frac{\partial \mu_{*}}{\partial c}\right|_{c_{e q}}\left(c(\Gamma)-c_{e q}\right), \quad V_{N}^{\Gamma}=\frac{\nu_{+} M_{+}}{\rho_{g}} k_{*} c_{e q} \frac{A_{N}}{R T}=\left.\frac{v_{+} \nu_{*} M_{+}}{\rho_{g} R T} k_{*} c_{e q} \frac{\partial \mu_{*}}{\partial c}\right|_{c_{e q}}\left(c(\Gamma)-c_{e q}\right)
$$

Further we assume that $\mu_{*}$ is a chemical potential of an ideal gas, $M_{*} \mu_{*}=\eta(T)+R T \ln c$. Then not far from the chemical equilibrium

$$
V_{N}^{\Gamma}=\frac{k_{*} v_{+} \nu_{*} M_{+}}{\rho_{g}}\left(c(\Gamma)-c_{e q}\right)
$$

In the small strain approach the equation for $c_{e q}(5)$ takes the form

$$
h A_{N}=\boldsymbol{\sigma}:[\boldsymbol{\varepsilon}]-[f]+h v_{*}\left(\eta(T)+R T \ln c_{e q}\right)=0, \quad h=\frac{\rho_{0}}{v_{-} M_{-}}
$$

where $\boldsymbol{\sigma}$ and $\varepsilon$ Cauchy stress tensor and strain tensor, brackets $[\phi]=\phi_{+}-\phi_{-}$denote a jump. Free energy densities of solid constituents are represented by quadratic dependencies

$$
f_{ \pm}=f_{ \pm}^{0}(T)+\frac{1}{2}\left(\boldsymbol{\varepsilon}-\boldsymbol{\varepsilon}_{ \pm}^{c h}\right): \mathbf{C}_{ \pm}:\left(\boldsymbol{\varepsilon}-\boldsymbol{\varepsilon}_{ \pm}^{c h}\right)
$$

where $\boldsymbol{\varepsilon}_{-}^{c h}=0, \boldsymbol{\varepsilon}_{+}^{c h}=\varepsilon^{c h} \mathbf{E}$. Then the constitutive equation takes the form $\boldsymbol{\sigma}_{ \pm}=\mathbf{C}_{ \pm}:\left(\boldsymbol{\varepsilon}-\boldsymbol{\varepsilon}_{ \pm}^{c h}\right)$. Thus, to describe the chemical reaction front kinetic we have to find the equilibrium concentration $c_{e q}$ from the equation (7) taking into account the dependencies of the stresses on the chemical reaction front position, and to find the current concentration $c(\Gamma)$ at the chemical reaction front from the solution of the diffusion problem with boundary conditions $c=c_{0}$ at the outer surface of the body and the condition

$$
D \mathbf{N} \cdot \nabla c+k_{*} \nu_{*}\left(c-c_{e q}\right)=0
$$

at the chemical reaction front. The last condition is the mass balance at the reaction front not far from the chemical equilibrium.

\section{Axially symmetric problem}

As an example we consider two axially-symmetric problems: a linear-elastic cylinder of the radius $R$ under all-round external stress $\sigma_{0}$ and an infinite linear-elastic medium with a cylindrical hole of the radius $R$ under internal pressure $p$ and external stress $\sigma_{0}$ (Fig.2). Note that the comparison of the results allows us to understand the role of the surface curvature sign. We suppose that the oxidizing gas surrounds the cylinder and also contains inside the cylindrical hole. 

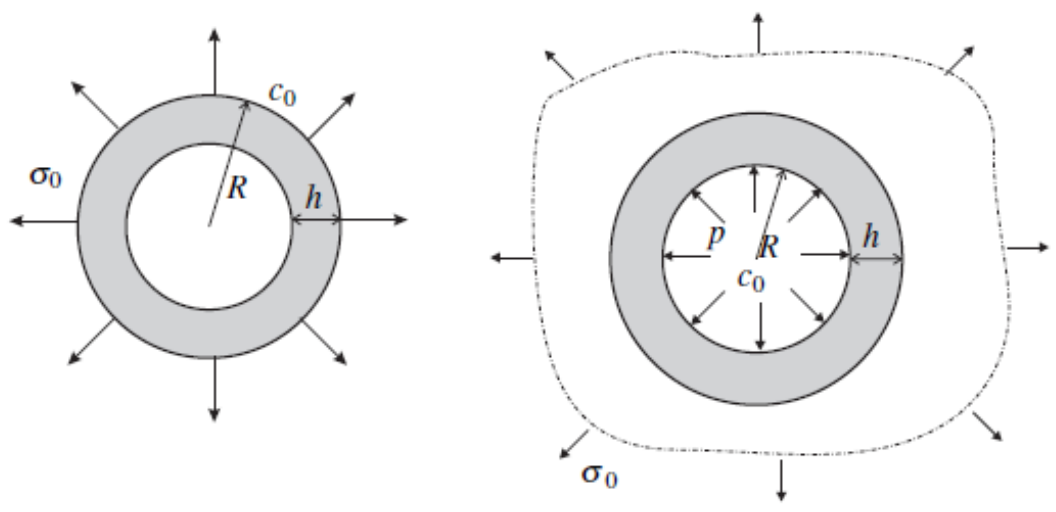

Fig.2. Axially symmetric problems: a cylinder and a cylindrical hole in infinite medium, $h$ is a thickness of the oxide layer

The dependencies of the oxide layer thickness on time in the cases of the cylinder and hole are presented in Fig.3. It is seen that the reaction front spreads faster in the case of the cylinder than in the case of the hole of the same radius. The curves for the cylinder and the hole are divided by the curve for the plane reaction front obtained as limit cases of the fronts in the cases of the cylinder and the hole at infinite radius.

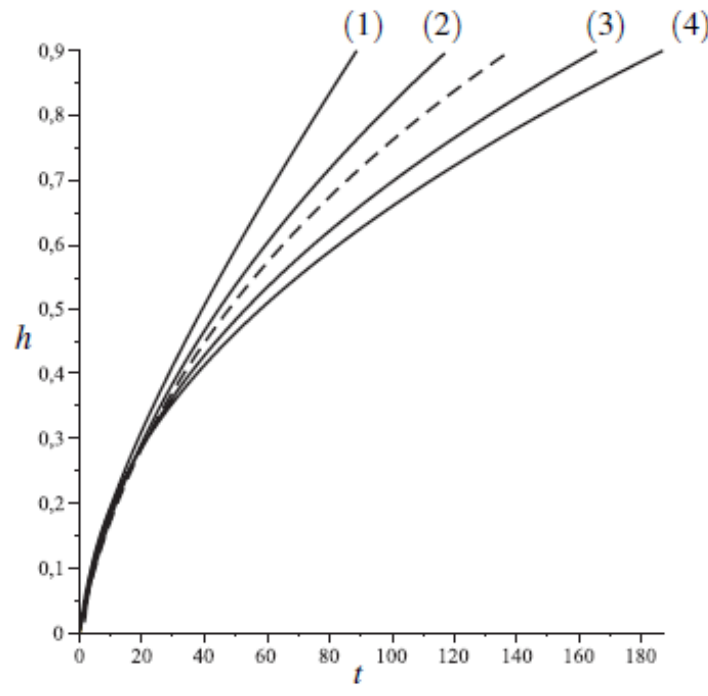

Fig.3. The dependencies of the oxide layer thickness on time (1) -- the cylinder $R=1$, (2) - the cylinder $R=2$, (3) - the hole $R=2$, (4) - the hole $R=1$, dashed line - the case of the plane reaction front (oxidation of the half-space)

If the reaction front moves then stresses at the front change and, thus, the equilibrium concentration changes. Fig. 4 shows the dependencies of the equilibrium concentration on the layer thickness at various external stresses. In the case of the cylinder the equilibrium concentration decreases if the oxide layer thickness increases. This in turn promotes the reaction front propagation.

In contrast, the oxide layer growth in the case of the hole leads to the increase of the equilibrium concentration, and this may block the reaction if $c(\Gamma)<c_{e q}$. Also $c(\Gamma)$ cannot be greater than the concentration $c_{0}$ at the hole surface. Thus, the inequality $c_{e q} / c_{0}<1$ is the necessary condition of the 
reaction front propagation. From Fig.4 (b) it is seen that internal stresses produced by the chemical reaction can block the reaction if the oxide layer thickness is more than a critical value $h_{c r}$. The value of the critical thickness depends on the external stress and increases at compression and decreases at tension. Thus, the sign of the applied stress affects the chemical reactions kinetics.

If the external stress is zero then, given the concentration $c_{0}$ and the energy parameter $\gamma=h \eta(T)-[f]$, internal stresses can block the reaction in the cylinder if the layer thickness is less than a critical value. In this case the equilibrium concentration at the front $c_{e q}>c_{0}$. Thus, an initial oxide layer on the cylinder surface may play a protective role, but its damage leads to further chemical reactions. Similar to the case of the hole in the infinite medium, the critical layer thickness increases at compression and decreases at tension. Thus, given initial thickness, tension may initiate further chemical reaction. On the other hand, compression may decelerate and even block the reaction front propagation.

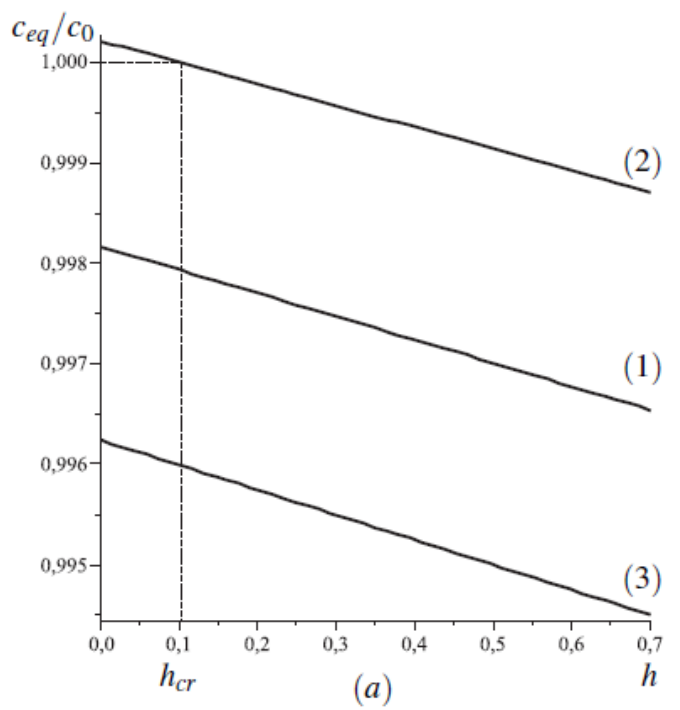

(a)

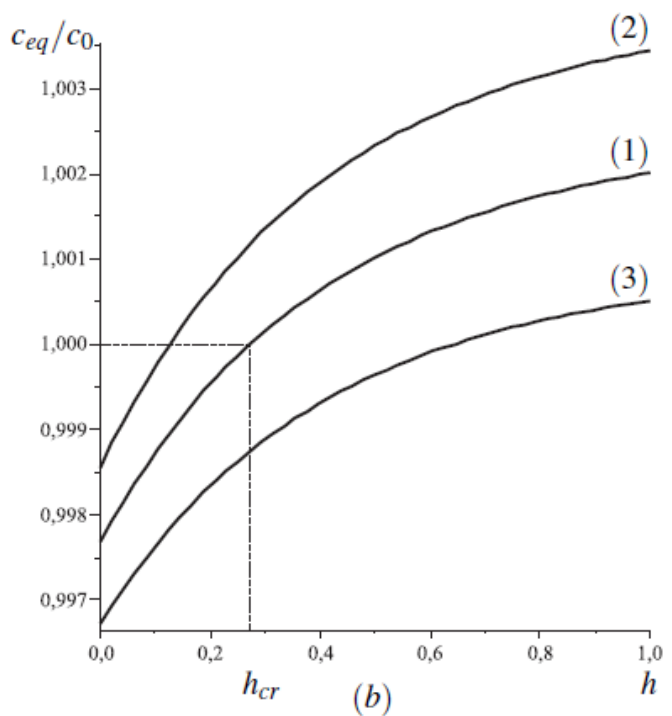

(b)

Fig.4. The dependence of the equilibrium concentration at the reaction front on the oxide layer thickness in the case of the cylinder (a) and the hole (b) at various external stresses: (1) $\sigma_{0}=0$, (2) $\sigma_{0}=0.1$, (3) $\sigma_{0}=-0.1$

\section{FEM simulation of chemical reaction front propagation}

Numerical realization of the model developed includes the following steps.

1. Find stresses $\sigma$ at the reaction front for a body with an oxide domain.

2. Find the equilibrium concentration $c_{e q}(\sigma, \mathbf{N}, T$,) at the reaction front solving the equation (7).

3. Solve the diffusion problem. Find $c(\Gamma)$ at the reaction front.

4. Calculate $V_{N}^{\Gamma}$.

5. Give increment $\Delta s_{N}=V_{N}^{\Gamma} \Delta t$ to the points of the reaction front and find a new position of the front.

6. Repeat the procedure.

An example of FEM-simulation of the evolution of the chemical reaction front propagation in a specimen with a semi-circular groove is shown in Fig. 5. 

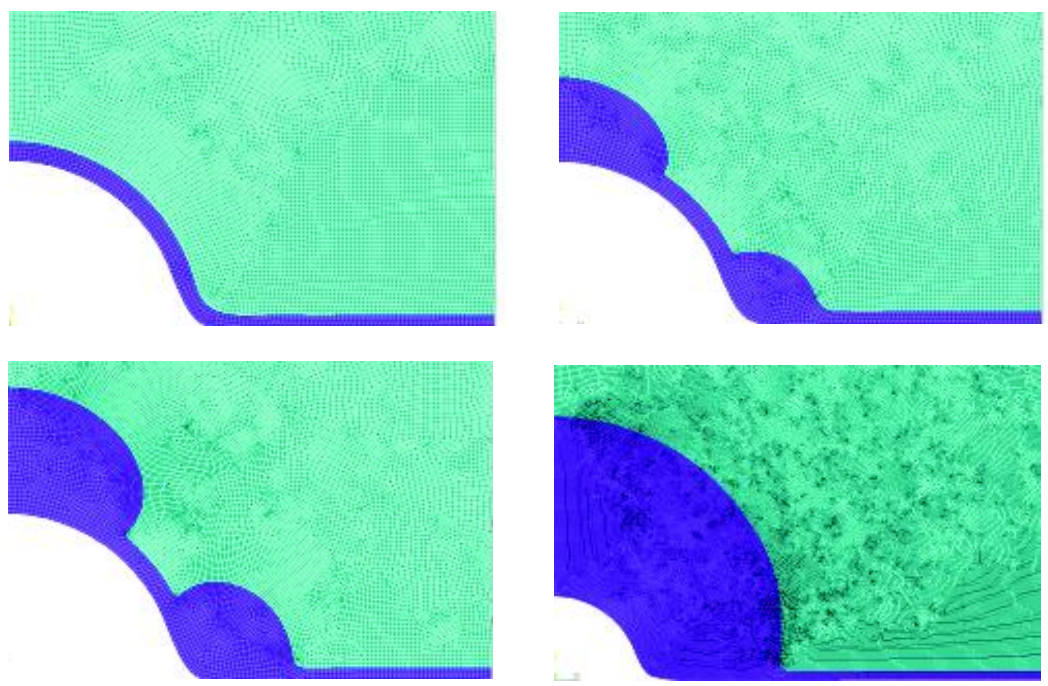

Fig. 5. Evolution of the chemical reaction front in the vicinity of a semi-circular groove under external horizontal tension (FEM-simulations made by Igor Korolev)

\section{Summary}

We developed a model for describing stress-assist chemical reaction front propagation. The model includes the expressions of the chemical transformations strains and chemical affinity tensor, and kinetic relationship that can be represented in terms of equilibrium and current concentrations of the gas constituents. The model shows how internal and external stresses affect the reaction kinetics and predicts the locking effects.

Acknowledgement. This work was supported by Russian Foundation for Basic Research (Grant 1001-00670), Sandia National Laboratories and RAS Programs for Fundamental Research.

\section{References}

[1] C.L. Muhlstein, S.B. Brown, R.O. Ritchie. Sensors and Actuators, Vol. A 94 (2001) p. 177188

[2] C.L. Muhlstein, E.A. Stach, R.O. Ritchie. J. Acta Materialia Vol. 50 (2002) p. 3579-3595

[3] S.T. Kelly, B.M. Clemens. J. Applied Physics 108 (2010) p. 013521

[4] E. Deal, A.S. Grove. J. Applied Physics. Vol. 36 (1965) p. 3770-3778.

[5] F. Larche, J.W. Cahn. Acta Metall. Vol. 21 (1973) p. 1051-1063.

[6] F. Larche, J.W. Cahn. Acta Metall. Vol. 26 (1978) p. 53-60.

[7] F. Larche, J.W. Cahn. Acta Metall. Vol. 26 (1978) p. 1579-1589.

[8] F. Larche, J.W. Cahn. 1982. Acta Metall. 30, (1978) p. 1835-1845.

[9] C. H. Wu. J. Mechanics and Physics of Solids. Vol. 49 (2001) p. $1771-1794$

[10] A.B.Freidin. In: Proceedings of XXXVI International Summer School-Conference APM' 2009. St. Petersburg. Institute for Problems in Mechanical Engineering, p. 231-237. http://apm-conf.spb.ru/2009/apm2009_proceedings.pdf.

[11] P. Glansdorff, I. Prigogine. Thermodynamic theory of structure, stability and fluctuations. Wiley-Interscience, London, NY, Sydney, Toronto. 